\title{
Model dependence of the neutron-skin thickness on the symmetry energy
}

\author{
C. Mondal, ${ }^{1,}$ B. K. Agrawal,,${ }^{1, \dagger}$ M. Centelles, ${ }^{2}$ G. Colò,${ }^{3,4}$ X. Roca-Maza, ${ }^{3,4}$ N. Paar, ${ }^{5}$ X. Viñas, ${ }^{2}$ S. K. Singh, ${ }^{6}$ and S. K. Patra ${ }^{6}$ \\ ${ }^{1}$ Saha Institute of Nuclear Physics, 1/AF Bidhannagar, Kolkata 700064, India \\ ${ }^{2}$ Departament d'Estructura i Constituents de la Matèria and Institut de Ciències del Cosmos, Facultat de Física, \\ Universitat de Barcelona, Diagonal 645, E-08028 Barcelona, Spain \\ ${ }^{3}$ Dipartimento di Fisica, Università degli Studi di Milano, via Celoria 16, I-20133 Milano, Italy \\ ${ }^{4}$ INFN, sezione di Milano, via Celoria 16, I-20133 Milano, Italy \\ ${ }^{5}$ Department of Physics, Faculty of Science, University of Zagreb, Zagreb, Croatia \\ ${ }^{6}$ Institute of Physics, Bhubhaneshwar, 751005, India
}

(Received 8 April 2016; published 2 June 2016)

\begin{abstract}
The model dependence in the correlations of the neutron-skin thickness in heavy nuclei with various symmetryenergy parameters is analyzed by using several families of systematically varied microscopic mean-field models. Such correlations show a varying degree of model dependence once the results for all the different families are combined. Some mean-field models associated with similar values of the symmetry-energy slope parameter at saturation density $L$, and pertaining to different families, yield a greater-than-expected spread in the neutron-skin thickness of the ${ }^{208} \mathrm{~Pb}$ nucleus. The effective value of the symmetry-energy slope parameter $L_{\text {eff }}$, determined by using the nucleon density profiles of the finite nucleus and the density derivative $S^{\prime}(\rho)$ of the symmetry energy starting from about saturation density up to low densities typical of the surface of nuclei, seems to account for the spread in the neutron-skin thickness for the models with similar $L$. The differences in the values of $L_{\text {eff }}$ are mainly due to the small differences in the nucleon density distributions of heavy nuclei in the surface region and the behavior of the symmetry energy at subsaturation densities.
\end{abstract}

DOI: 10.1103/PhysRevC.93.064303

\section{INTRODUCTION}

Terrestrial nuclei are mostly asymmetric (i.e., $N \neq Z$ ), except for the light nuclei with proton number $Z \leqslant 28$. At the other extreme, matter in compact astrophysical objects such as neutron stars is highly asymmetric [1]. The asymmetry in the finite nuclei primarily arises due to the balance between the Coulomb energy and the nuclear symmetry energy. The conditions of $\beta$ equilibrium and charge neutrality render matter in a neutron star highly asymmetric or predominantly composed of neutrons [2]. The densities at the center of nuclei are close to the normal saturation density $\rho_{0}\left(0.16 \mathrm{fm}^{-3}\right)$, whereas the densities at the center of neutron stars are predicted to be typically a few times $\rho_{0}$. Thus, accurate knowledge of the nuclear symmetry energy over a wide range of densities is indispensable to understand a variety of phenomena in finite nuclei as well as in neutron stars.

The details of the density dependence of the nuclear symmetry energy remain hard to isolate, although progress in this direction has been made in the last few years (see, for instance, Refs. [3-20] and the experimental and theoretical works quoted therein). The density dependence of the nuclear symmetry energy around saturation is governed to leading order by its density derivative, which is expressed as

$$
L=3 \rho_{0}\left(\frac{d S(\rho)}{d \rho}\right)_{\rho_{0}},
$$

\footnotetext{
*chiranjib.mondal@saha.ac.in

†bijay.agrawal@saha.ac.in
}

where $S(\rho)$ is the symmetry energy at density $\rho$. The macroscopic nuclear droplet model (DM) of Myers and Swiatecki [21,22] suggests that various symmetry-energy parameters and the neutron-skin thickness in a heavy nucleus are related to one another. The neutron-skin thickness is defined as the difference between the rms radii for the density distributions of the neutrons and protons in the nucleus:

$$
\Delta r_{\mathrm{np}} \equiv\left\langle r^{2}\right\rangle_{n}^{1 / 2}-\left\langle r^{2}\right\rangle_{p}^{1 / 2}
$$

Nuclear mean-field models predict a nearly linear correlation of $\Delta r_{\mathrm{np}}$ of a heavy nucleus such as ${ }^{208} \mathrm{~Pb}$ with the slope of the equation of state of neutron matter at a subsaturation density around $0.1 \mathrm{fm}^{-3}[23,24]$, with the density derivative of the symmetry energy $L[3,4,6,7,16,25,26]$, and with the surface symmetry energy in a finite nucleus $[4,6,27]$. The correlation of a finite nucleus property such as $\Delta r_{\text {np }}$ with a bulk property of infinite nuclear matter such as $L$ can be interpreted as basically due to the dependence of $\Delta r_{\mathrm{np}}$ on the surface symmetry energy. In a local density approximation the surface symmetry energy can be correlated with $L$, and this fact therefore implies the correlation between $\Delta r_{\text {np }}$ and $L$. Macroscopic approaches such as the DM $[21,22]$ often provide insightful guidance into the global features of many of these correlations [6,7,14], as is briefly recalled in the next section.

The Lead Radius Experiment (PREX) [28,29] has recently measured the neutron skin thickness $\Delta r_{\mathrm{np}}$ of ${ }^{208} \mathrm{~Pb}$. This experiment is performed via parity-violating electron scattering [30] and provides the first purely electroweak, model-independent measurement of the weak charge form factor, closely connected to the neutron distribution of the ${ }^{208} \mathrm{~Pb}$ nucleus [30]. By measuring the weak form factor of ${ }^{208} \mathrm{~Pb}$ at momentum transfer $q \approx 0.475 \mathrm{fm}^{-1}$, PREX was able to determine 
$\Delta r_{\mathrm{np}}=0.33_{-0.18}^{+0.16} \mathrm{fm}$ [29]. Recently, a followup measurement of PREX has been proposed which intends to measure the neutron-skin thickness in the ${ }^{208} \mathrm{~Pb}$ nucleus with an accuracy of $0.06 \mathrm{fm} \mathrm{[31].} \mathrm{Hadronic} \mathrm{probes} \mathrm{are} \mathrm{also} \mathrm{used}$ to estimate the neutron distribution in nuclei [32-36]. In this case, the strong interaction needs to be modeled and, therefore, deducing the neutron radius from these experiments can imply various theoretical uncertainties, which in some cases are difficult to estimate. The analyses from recent hadronic experiments have led to varying values of the neutron-skin thickness of ${ }^{208} \mathrm{~Pb}: \Delta r_{\mathrm{np}}=0.16 \pm$ 0.02 (stat) \pm 0.04 (syst) fm [35] and $\Delta r_{\mathrm{np}}=0.211_{-0.063}^{+0.054} \mathrm{fm}$ [33]. A very recent measurement of coherent pion photoproduction [37] provides a value $\Delta r_{\mathrm{np}}=0.15 \pm 0.03 \mathrm{fm}$ for ${ }^{208} \mathrm{~Pb}$. Also, a neutron-skin-thickness $\Delta r_{\mathrm{np}}=0.165 \pm$ 0.009 (expt) \pm 0.013 (theor) \pm 0.021 (est) fm has been extracted recently by comparing theory with the measured electric dipole polarizability in ${ }^{208} \mathrm{~Pb}$ [10,19,38-40].

Ongoing efforts are underway to perform an accurate and model-independent measurement of the neutron-skin thickness in the ${ }^{208} \mathrm{~Pb}$ nucleus. At the same time, it may not be straightforward for theory to extract various symmetryenergy parameters from the neutron-skin thickness in a modelindependent fashion. Starting from the seminal papers of more than a decade ago $[3,23,24,41]$, the focus has mainly been on the linear correlation between the neutron-skin thickness and the slope parameter $L$ of the symmetry energy. The correlation is satisfied to a large degree in the microscopic calculations with mean-field models but it is not perfect and a certain model dependence appears in the results (see for example the plots in Refs. $[3,4,6,7,14,24,41])$. By model dependence we mean here that different mean-field models may predict similar values for the $L$ parameter but different neutron-skin thickness in a heavy nucleus. This may be seen, for example, from Fig. 2 and Table II of Ref. [14], some models deviate from the linear correlation. This analysis was done by using different unbiasedly selected mean-field models. We would like to complement the earlier analysis with one based on families of systematically varied models, in an attempt to identify the sources for the model dependence in the correlations.

In the present work we revisit the correlations of $\Delta r_{\mathrm{np}}$ with various symmetry-energy parameters. The plausible causes for the existence of a model dependence in these correlations are investigated. The correlations are evaluated by using five different families of systematically varied microscopic mean-field models. Three out of these five families correspond to relativistic energy density functionals $[42,43]$ and the remaining two families correspond to a nonrelativistic functional [44]. We also predict the neutron-skin thickness of the neutron-rich nucleus ${ }^{132} \mathrm{Sn}$ which has not been measured yet.

The paper is organized as follows: The geometrical definitions employed to decompose the neutron-skin thickness into bulk and surface contributions $[14,45]$ are briefly outlined in Sec. II. We also provide in this section some results derived from the macroscopic DM suggesting possible connections between the neutron-skin thickness and various symmetryenergy parameters. In Sec. III, the results for the correlations of the neutron-skin thickness in the ${ }^{208} \mathrm{~Pb}$ and ${ }^{132} \mathrm{Sn}$ nuclei with the symmetry-energy parameters obtained for several families of the systematically varied models are presented. The plausible causes for the model dependence in such correlations are investigated in detail. The main conclusions are presented in Sec. IV.

\section{NEUTRON-SKIN THICKNESS AND SYMMETRY-ENERGY PARAMETERS}

From a geometrical point of view, the neutron-skin thickness in a nucleus may be thought to originate from two different effects: One effect is due to the separation between the mean sharp surfaces of the neutron- and proton-density distributions. Since this effect corresponds to a different extent of the bulk region of the neutron and proton densities, we refer to it as the bulk contribution to the neutron-skin thickness. The other effect is due to the different surface widths of the neutron and proton densities, which we call the surface contribution to the neutron-skin thickness. To compute the bulk and surface contributions to the neutron-skin thickness in a nucleus requires a proper definition of these quantities based on the nuclear densities. In this respect we follow closely the method described by Hasse and Myers [46] and which we applied in Refs. [14,45].

To determine the position of the neutron and proton effective surfaces one can define different radii. In particular, one can define the central radius $C$ as

$$
C=\frac{1}{\rho(0)} \int_{0}^{\infty} \rho(r) d r .
$$

Another option for the mean position of the surface is the equivalent radius $R$, which is the radius of a uniform sharp distribution whose density equals the bulk value of the actual density and has the same number of particles:

$$
\frac{4}{3} \pi R^{3} \rho(\text { bulk })=4 \pi \int_{0}^{\infty} \rho(r) r^{2} d r .
$$

Finally, one can also define the equivalent rms radius $Q$ that describes a uniform sharp distribution with the same rms radius as the given density:

$$
\frac{3}{5} Q^{2}=\left\langle r^{2}\right\rangle
$$

The radii $C, R$, and $Q$ are related by the expressions [46]

$$
Q=R\left(1+\frac{5}{2} \frac{b^{2}}{R^{2}}+\cdots\right), \quad C=R\left(1-\frac{b^{2}}{R^{2}}+\cdots\right),
$$

where $b$ is the surface width of the density profile and is defined as

$$
b^{2}=-\frac{1}{\rho(0)} \int_{0}^{\infty}(r-C)^{2} \frac{d \rho(r)}{d r} d r,
$$

which provides a measure of the extent of the surface of the nucleus. The neutron-skin thickness, which is defined through the rms radii, can be expressed by

$$
\Delta r_{n p}=\sqrt{\frac{3}{5}}\left(Q_{n}-Q_{p}\right)
$$


and, by using Eq. (6), reads

$$
\Delta r_{\mathrm{np}}=\sqrt{\frac{3}{5}}\left[\left(R_{n}-R_{p}\right)+\frac{5}{2}\left(\frac{b_{n}^{2}}{R_{n}}-\frac{b_{p}^{2}}{R_{p}}\right)\right],
$$

which clearly separates the bulk and surface contributions as

$$
\Delta r_{\mathrm{np}}^{\mathrm{bulk}} \equiv \sqrt{\frac{3}{5}}\left(R_{n}-R_{p}\right)
$$

and

$$
\Delta r_{\mathrm{np}}^{\mathrm{surf}} \equiv \sqrt{\frac{3}{5}} \frac{5}{2}\left(\frac{b_{n}^{2}}{R_{n}}-\frac{b_{p}^{2}}{R_{p}}\right) .
$$

In Eqs. (9) and (11), we have neglected $O\left[b^{4} / R^{3}\right]$ and higherorder terms since they represent a small correction [14] to $\Delta r_{\mathrm{np}}$ - of less than or around $1 \%-2 \%$ - that will leave our conclusions unchanged.

To extract the bulk and surface contributions to the neutronskin thickness from the quantal proton and neutron densities obtained within the Skyrme Hartree-Fock or the relativistic mean-field models, we proceed as in Refs. [14,45]. That is, we fit the self-consistent quantal proton and neutron densities by two-parameter Fermi $(2 \mathrm{pF})$ distributions

$$
\rho_{q}(r)=\frac{\rho_{0, q}}{1+\exp \left[\left(r-C_{q}\right) / a_{q}\right]},
$$

where $q=n, p$. The parameters $\rho_{0, q}, C_{q}$, and $a_{q}$ are adjusted to reproduce the nucleon numbers as well as the values for the second and fourth moments of the actual density distributions, i.e., $\left\langle r_{q}^{2}\right\rangle$ and $\left\langle r_{q}^{4}\right\rangle$. Once this fit is done, we can express Eqs. (9)(11) for the neutron-skin thickness in terms of the parameters $C_{q}$ and $a_{q}$, taking into account Eq. (6) and the fact that, for a $2 \mathrm{pF}$ distribution, $b=\pi a / \sqrt{3}$. Therefore, the bulk and surface contributions to the neutron-skin thickness can be written as

$$
\begin{aligned}
\Delta r_{\mathrm{np}}^{\mathrm{bulk}} & =\sqrt{\frac{3}{5}}\left[\left(C_{n}-C_{p}\right)+\frac{\pi^{2}}{3}\left(\frac{a_{n}^{2}}{C_{n}}-\frac{a_{p}^{2}}{C_{p}}\right)\right], \\
\Delta r_{\mathrm{np}}^{\mathrm{surf}} & =\sqrt{\frac{3}{5} \frac{5 \pi^{2}}{6}\left(\frac{a_{n}^{2}}{C_{n}}-\frac{a_{p}^{2}}{C_{p}}\right),}
\end{aligned}
$$

up to terms of order $O\left[a^{4} / C^{3}\right]$. It should be mentioned that the $\Delta r_{\mathrm{np}}$ values calculated from the actual densities obtained selfconsistently match very well with those calculated by summing Eqs. (13) and (14) after applying our prescription to determine the parameters of the Fermi function.

Some insight about possible correlations between the neutron-skin thickness and different observables related to the symmetry energy is provided by the DM [22]. Within this model, which neglects shell-correction effects, the neutronskin thickness is expressed by

$$
\Delta r_{\mathrm{np}}=\sqrt{\frac{3}{5}}\left[t-\frac{e^{2} Z}{70 J}+\frac{5}{2 R}\left(b_{n}^{2}-b_{p}^{2}\right)\right],
$$

where $e^{2} Z / 70 \mathrm{~J}$ is a correction due to the Coulomb interaction, $R=r_{0} A^{1 / 3}$ is the nuclear radius, and $b_{n}$ and $b_{p}$ are the surface widths of the neutron- and proton-density profiles. The quantity $t$ in Eq. (15) represents the distance between the location of the neutron and proton mean surfaces and therefore is proportional to the bulk contribution to the neutron-skin thickness. In the DM its value is given by

$$
t=\frac{3}{2} r_{0} \frac{J}{Q_{\text {stiff }}} \frac{I-I_{C}}{1+x_{A}},
$$

with

$$
I_{C}=\frac{3 e^{2}}{5 r_{0}} \frac{Z}{12 J} A^{-1 / 3} \quad \text { and } x_{A}=\frac{9 J}{4 Q_{\text {stiff }}} A^{-1 / 3},
$$

where $I=(N-Z) / A, J$ is the bulk symmetry energy at saturation, and $Q_{\text {stiff }}$ is the surface stiffness. For each meanfield model, the parameters $r_{0}$ and $J$ can be obtained from calculations in infinite nuclear matter and $Q_{\text {stiff }}$ from calculations performed in semi-infinite nuclear matter $[7,47,48]$.

Within the DM, the symmetry-energy coefficient of a finite nucleus of mass number $A$ is given by

$$
a_{\text {sym }}(A)=\frac{J}{1+x_{A}} .
$$

Replacing $a_{\text {sym }}(A)$ in Eq. (16), the separation distance between the mean surfaces of neutrons and protons can be recast as

$$
t=\frac{2 r_{0}}{3 J}\left[J-a_{\mathrm{sym}}(A)\right] A^{1 / 3}\left(I-I_{C}\right)
$$

The link between a property in finite nuclei such as $a_{\text {sym }}(A)$ and some symmetry-energy parameters in infinite nuclear matter may be obtained from the observation [6] that, for a heavy nucleus, there is a subsaturation density, which for ${ }^{208} \mathrm{~Pb}$ is around $0.1 \mathrm{fm}^{-3}$, such that the symmetry-energy coefficient in the finite nucleus $a_{\text {sym }}(A)$ equals the symmetry energy in nuclear matter $S(\rho)$ computed at that density. This relation is roughly independent of the mean-field model used to compute it. Around the saturation density $\rho_{0}$ the symmetry energy can be expanded as

$$
S(\rho) \simeq J-L\left(\frac{\rho_{0}-\rho}{3 \rho_{0}}\right)+\frac{1}{2} K_{\mathrm{sym}}\left(\frac{\rho_{0}-\rho}{3 \rho_{0}}\right)^{2} .
$$

Consequently, the distance $t$ can be finally expressed approximately as [6]

$$
t=\frac{2 r_{0}}{3 J} L\left(\frac{\rho-\rho_{0}}{3 \rho_{0}}\right)\left[1-\frac{K_{\mathrm{sym}}}{2 L}\left(\frac{\rho-\rho_{0}}{3 \rho_{0}}\right)\right] A^{1 / 3}\left(I-I_{C}\right) .
$$

Equations (19) and (21) suggest correlations between the bulk neutron-skin thickness in finite nuclei and some isovector indicators such as $J-a_{\text {sym }}(A), a_{\text {sym }}(A) / J$, and $L$, which is discussed in detail in this paper. To compute the average symmetry energy of a finite nucleus with the DM [Eq. (18)] requires knowledge of the surface stiffness $Q_{\text {stiff }}$, which in turn requires semi-infinite nuclear-matter calculations [7]. An efficient procedure to circumvent this is to evaluate $a_{\text {sym }}(A)$ within a local density approximation as [9]

$$
a_{\mathrm{sym}}(A)=\frac{4 \pi}{A I^{2}} \int\left[r^{2} \rho(r) I^{2}(r)\right] S(\rho(r)) d r
$$

where $I(r)=\left[\rho_{n}(r)-\rho_{p}(r)\right] / \rho(r)$ is the local isospin asymmetry and $\rho(r)$ is the sum of the neutron and proton densities. This approximation works very well for medium-heavy ${ }^{132} \mathrm{Sn}$ or heavy ${ }^{208} \mathrm{~Pb}$ nuclei [49]. 


\section{RESULTS AND DISCUSSIONS}

The neutron-skin thickness and several symmetry-energy parameters are calculated by using five different families of systematically varied models, namely, the SAMi-J [10,50], DDME [51], FSV, TSV, and KDE0-J models. The energy density functional associated with DDME, FSV, and TSV corresponds to an effective Lagrangian density typical of the relativistic mean-field models, whereas SAMi-J and KDE0-J are based on the standard form of the Skyrme force.

We obtained the different families of systematically varied parameter sets so that they explore different values of the symmetry-energy parameters around an optimal value, while reasonably keeping the quality of the best fit. The values of the neutron-skin thickness in a heavy nucleus like ${ }^{208} \mathrm{~Pb}$ vary over a wide range within the families due to the variations of the symmetry-energy parameters. The parameter sets for the FSV, TSV, and KDE0-J families are obtained in the present work. The effective Lagrangian density employed for the FSV family is similar to that for the FSU model [52]. In addition to the coupling of $\rho$ meson to the nucleons as conventionally employed, the presence of a cross coupling between the $\omega$ and $\rho$ mesons in the FSU model enables one to vary the symmetry energy and, accordingly, the symmetry-energy slope parameter $L$ over a wide range without significantly affecting the quality of the fit to the bulk properties of the finite nuclei. The TSV family is obtained by using the effective Lagrangian density as introduced in Ref. [53] in which the $\rho$ meson and its coupling to the $\sigma$ meson govern the isovector part of the interactions between the nucleons. The $\omega$ - $\rho$ cross coupling in the FSV family and the $\sigma-\rho$ cross coupling in the TSV family produce different behavior in the density dependence of the symmetry energy, because the source term for the $\omega$ field is governed by the baryon density and that for the $\sigma$ field is governed by the scalar density. The experimental data employed to determine the TSV and FSV families are the total binding energies for the ${ }^{16} \mathrm{O},{ }^{40,48} \mathrm{Ca},{ }^{68} \mathrm{Ni},{ }^{90} \mathrm{Zr},{ }^{100,132} \mathrm{Sn}$, and ${ }^{208} \mathrm{~Pb}$ nuclei, and the root mean square charge radii for the ${ }^{16} \mathrm{O}$, ${ }^{40,48} \mathrm{Ca},{ }^{90} \mathrm{Zr}$, and ${ }^{208} \mathrm{~Pb}$ nuclei. The energy density functional for the KDE0-J family calculated within the Skyrme ansatz is taken from the KDE0 force of Ref. [54]. The model parameters are constrained to yield the nuclear matter incompressibility coefficient in the range of $225-250 \mathrm{MeV}$. The calculated values of the total binding energy and the charge radius for the ${ }^{208} \mathrm{~Pb}$ nucleus obtained for all the models considered deviate from the experimental data only within $0.25 \%$ and $0.8 \%$, respectively.

\section{A. Correlation plots associated with isovector indicators}

As we discussed in the previous section, the DM is a useful guideline to suggest the kind of correlations that we can expect between the neutron-skin thickness and the symmetry-energy parameters. As shown in Ref. [14], these correlations are mainly due to the bulk term of Eq. (15) rather than to the surface contribution to $\Delta r_{\mathrm{np}}$. In the bulk part of $\Delta r_{\mathrm{np}}$, the quantity $\left[J-a_{\text {sym }}(A)\right] / J$ determines the ratio of the surfacesymmetry-to-volume-symmetry energies, see Eq. (19); the close relation of different isovector observables in finite nuclei with the ratio of the surface to volume symmetry energies has

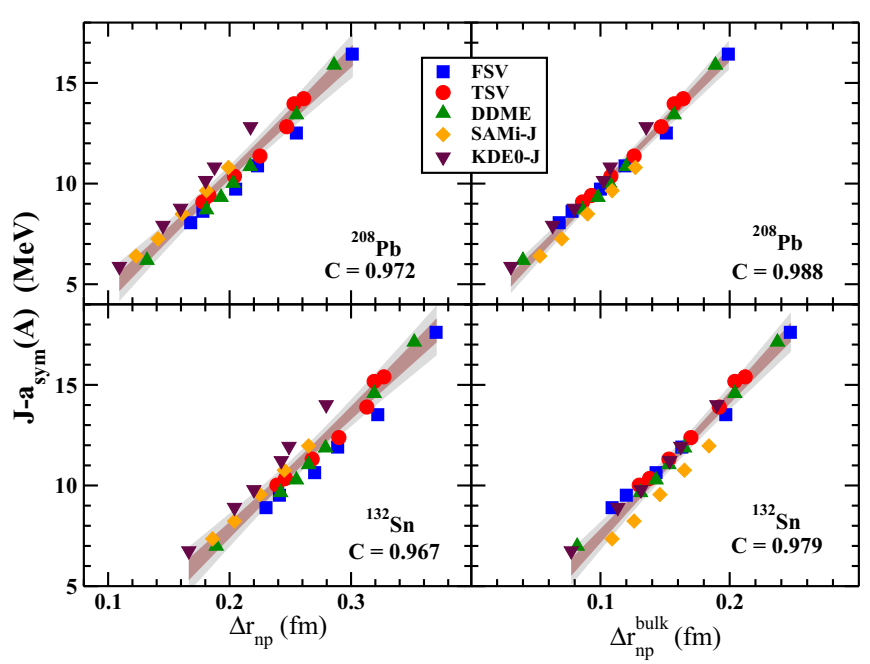

FIG. 1. Plots for the difference between the symmetry-energy coefficient for infinite nuclear matter $J$ and that for finite nuclei $a_{\text {sym }}(A)$ as a function of the neutron-skin thickness (left panels) and of the bulk part of the neutron-skin thickness (right panels). The results are obtained by using five different families of mean-field models; namely, FSV (blue squares), TSV (red circles), DDME (green triangles), SAMi-J (orange diamonds), and KDE0-J (maroon inverted triangles). The correlation coefficients are $C\left(J-a_{\mathrm{sym}}(A), \Delta r_{\mathrm{np}}\right)=$ $0.972(0.967)$ and $C\left(J-a_{\text {sym }}(A), \Delta r_{\text {np }}^{\text {bulk }}\right)=0.988(0.979)$ for ${ }^{208} \mathrm{~Pb}$ $\left({ }^{132} \mathrm{Sn}\right)$ nuclei. The inner (outer) colored regions depict the loci of the $95 \%$ confidence (prediction) bands of the regression (see, e.g., Chap. 3 of Ref. [55]).

been observed in several studies; cf., for example, Refs. [11,27] and references therein. The values of $r_{0}$ for the various models considered in the present work display only a small variation indicating that the total neutron-skin thickness $\Delta r_{\text {np }}$ of a given heavy nucleus may be correlated to the ratio $\left[J-a_{\text {sym }}(A)\right] / J$, or also to the difference $J-a_{\text {sym }}(A)$ provided the value of $J$ does not show a large variation as compared with $J-a_{\text {sym }}(A)$.

In Fig. 1, we plot for the ${ }^{208} \mathrm{~Pb}$ and ${ }^{132} \mathrm{Sn}$ nuclei the values of $J-a_{\text {sym }}(A)$ as a function of $\Delta r_{\text {np }}$ in the left panel, and as a function of the bulk part of the neutron-skin thickness $\Delta r_{\mathrm{np}}^{\text {bulk }}$ in the right panel. The results are reported for the five different families of systematically varied models; namely, FSV, TSV, SAMi-J, DDME, and KDE0-J, as indicated in the figure. Fairly evident linear correlations are observed between $J-$ $a_{\text {sym }}(A)$ and both $\Delta r_{\text {np }}$ and $\Delta r_{\text {np }}^{\text {bulk }}$. More quantitatively, if we calculate the Pearson's correlation coefficients $C(X, Y)$ [56], their values are $C\left(J-a_{\text {sym }}(A), \Delta r_{\mathrm{np}}\right)=0.972(0.967)$ and $C\left(J-a_{\text {sym }}(A), \Delta r_{\text {np }}^{\text {bulk }}\right)=0.988(0.979)$ for the ${ }^{208} \mathrm{~Pb}\left({ }^{132} \mathrm{Sn}\right)$ nuclei, respectively. Thus, the correlation of $J-a_{\text {sym }}(A)$ with $\Delta r_{\mathrm{np}}^{\text {bulk }}$ is a little higher than with $\Delta r_{\mathrm{np}}$ for both ${ }^{208} \mathrm{~Pb}$ and ${ }^{132} \mathrm{Sn}$ nuclei, as may be expected from the discussions in Sec. II.

Following Eq. (19), one can directly correlate [ $J-$ $\left.a_{\text {sym }}(A)\right] / J$ [or, equivalently, $a_{\text {sym }}(A) / J$ ] with $\Delta r_{\text {np }}$ of a heavy nucleus. In Fig. 2 we display the ratio $a_{\mathrm{sym}}(A) / J$ as a function of $\Delta r_{\mathrm{np}}$ and of $\Delta r_{n p}^{\text {bulk }}$ for the ${ }^{208} \mathrm{~Pb}$ and ${ }^{132} \mathrm{Sn}$ nuclei. The correlations of $a_{\text {sym }}(A) / J$ with $\Delta r_{\text {np }}$ are relatively weaker in comparison to those with $\Delta r_{\mathrm{np}}^{\text {bulk }}$. In the case of $a_{\text {sym }}(A) / J$ and $\Delta r_{\mathrm{np}}$ the correlation coefficient is $\left|C\left(a_{\mathrm{sym}}(A) / J, \Delta r_{\mathrm{np}}\right)\right|=$ 


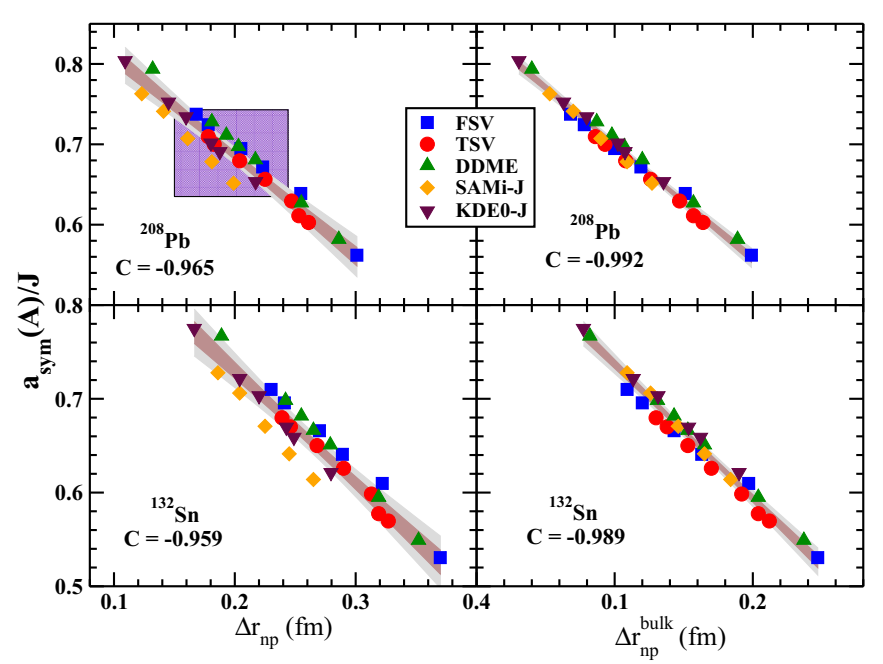

FIG. 2. Plots for the ratio of the nuclear symmetry-energy coefficient for finite nuclei $a_{\mathrm{sym}}(A)$ to that for infinite nuclear matter $J$, as a function of the neutron-skin thickness (left panels) and of the bulk part of the neutron-skin thickness (right panels). The square shaded region in the upper-left panel corresponds to $a_{\text {sym }}(A)=22.4 \pm$ $0.3 \mathrm{MeV}$ and $J=32.5 \pm 2.5 \mathrm{MeV}$. The correlation coefficients are $\left|C\left(a_{\text {sym }}(A) / J, \Delta r_{\text {np }}\right)\right|=0.965(0.959)$ and $\left|C\left(a_{\text {sym }}(A) / J, \Delta r_{\text {np }}^{\text {bulk }}\right)\right|=$ $0.992(0.989)$ for ${ }^{208} \mathrm{~Pb}\left({ }^{132} \mathrm{Sn}\right)$ nuclei. The inner (outer) colored regions depict the loci of the $95 \%$ confidence (prediction) bands of the regression (see, e.g., Chap. 3 of Ref. [55]).

0.965 (0.959) for ${ }^{208} \mathrm{~Pb}\left({ }^{132} \mathrm{Sn}\right)$, whereas in the case of $a_{\text {sym }}(A) / J$ and $\Delta r_{\text {np }}^{\text {bulk }}$ the correlation coefficient increases up to high values $\left|C\left(a_{\text {sym }}(A) / J, \Delta r_{\mathrm{np}}^{\text {bulk }}\right)\right|=0.992$ (0.989) for ${ }^{208} \mathrm{~Pb}\left({ }^{132} \mathrm{Sn}\right)$.

At this point, it is interesting to address the constraints on the neutron-skin thickness that may be deduced from the present study. The rectangular shaded region in the upper-left panel of Fig. 2 corresponds to $a_{\text {sym }}(A)=22.4 \pm$ $0.3 \mathrm{MeV}$ for ${ }^{208} \mathrm{~Pb}$ [57] and $J=32.5 \pm 2.5 \mathrm{MeV}$, which yields $\Delta r_{\mathrm{np}}=0.197 \pm 0.047 \mathrm{fm}$ in the ${ }^{208} \mathrm{~Pb}$ nucleus. The constraint $a_{\text {sym }}(A)=22.4 \pm 0.3 \mathrm{MeV}$ was evaluated in Ref. [57] by using the experimental binding-energy differences. Furthermore, the effect of the Coulomb interaction on the surface asymmetry and the effect of the surface diffuseness on the Coulomb energy were taken into account. The value of $J=32.5 \pm 2.5 \mathrm{MeV}$ as used in the present work has a quite reasonable overlap with those extracted either from a version of the finite-range droplet model (FRDM) that performs very well in reproducing the experimental mass systematics [58], by analyzing the experimental data on the electric dipole polarizability in ${ }^{68} \mathrm{Ni}$, ${ }^{120} \mathrm{Sn}$, and ${ }^{208} \mathrm{~Pb}$ nuclei [19], from specific manipulation of the semi-empirical mass formula [59], through analysis of the properties of semi-infinite nuclear matter [60], or by analyzing pygmy dipole resonance data on ${ }^{68} \mathrm{Ni}$ and ${ }^{132} \mathrm{Sn}$ nuclei [61]. This value of $J$ also overlaps with the conclusions provided in recent papers $[13,62]$.

It is desirable to check the degree of consistency between the results for different heavy nuclei, in particular between ${ }^{208} \mathrm{~Pb}$ and ${ }^{132} \mathrm{Sn}$ which would allow to predict the neutron-skin thickness of the nucleus ${ }^{132} \mathrm{Sn}$ assumed that the one of ${ }^{208} \mathrm{~Pb}$

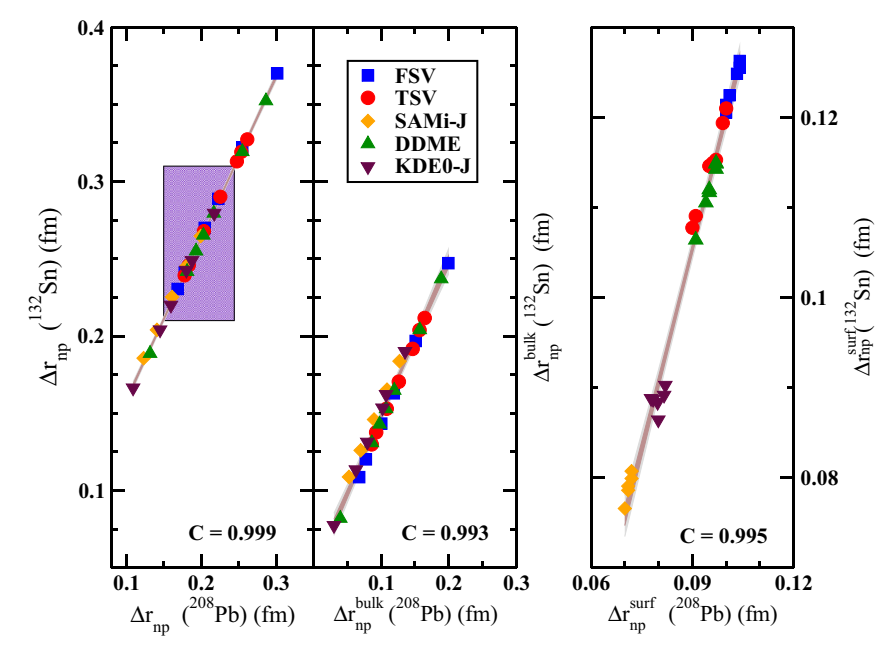

FIG. 3. Neutron-skin thickness (left) and its bulk (middle), and surface (right) contributions for the ${ }^{132} \mathrm{Sn}$ nucleus plotted against the same quantities for the ${ }^{208} \mathrm{~Pb}$ nucleus. The shaded region corresponds to the values of the neutron-skin thickness in ${ }^{132} \mathrm{Sn}$ determined from those estimated for the ${ }^{208} \mathrm{~Pb}$ nucleus (see also Fig. 2). The correlation coefficients obtained for the results presented in the left, middle, and right panels are $0.999,0.993$, and 0.995 , respectively. The inner (outer) colored regions depict the loci of the $95 \%$ confidence (prediction) bands of the regression (see, e.g., Chap. 3 of Ref. [55]).

is known. In the left panel of Fig. 3, we plot $\Delta r_{\mathrm{np}}$ for the ${ }^{132} \mathrm{Sn}$ nucleus against that for the ${ }^{208} \mathrm{~Pb}$ nucleus. Similarly, the results for $\Delta r_{\mathrm{np}}^{\text {bulk }}$ and $\Delta r_{\mathrm{np}}^{\text {surf }}$ are plotted in the middle and right panels of Fig. 3, respectively. It is observed that the values of $\Delta r_{\mathrm{np}}, \Delta r_{\mathrm{np}}^{\text {bulk }}$, and $\Delta r_{\mathrm{np}}^{\text {surf }}$ for the ${ }^{132} \mathrm{Sn}$ nucleus are very well correlated with the corresponding values in the ${ }^{208} \mathrm{~Pb}$ nucleus. This is in harmony with earlier work [8]. Hence, the information provided by the neutron skin of two heavy nuclei on the isovector channel of the nuclear effective interaction is mutually inclusive. Such an observation allows us to predict $\Delta r_{\mathrm{np}}=0.260 \pm 0.050 \mathrm{fm}$ for the ${ }^{132} \mathrm{Sn}$ nucleus by using the above estimated value for ${ }^{208} \mathrm{~Pb}$ of $\Delta r_{\mathrm{np}}=0.197 \pm 0.047 \mathrm{fm}$.

As recalled above and discussed in the literature (cf., in particular, Ref. [14] and references therein), we expect that the correlation between the neutron-skin thickness and $\left[J-a_{\text {sym }}(A)\right] / J$ leads to a correlation between the neutronskin thickness and the symmetry-energy slope parameter $L$. In Fig. 4, we display the variation of $L$ as a function of $\Delta r_{\mathrm{np}}$ (left panel), $\Delta r_{\mathrm{np}}^{\text {bulk }}$ (middle panel), and $\Delta r_{\mathrm{np}}^{\text {surf }}$ (right panel) for the ${ }^{208} \mathrm{~Pb}$ nucleus in the analyzed families of models. By using the constraint on $\Delta r_{\mathrm{np}}\left({ }^{208} \mathrm{~Pb}\right)$ obtained in Fig. 2, the bound on the value of $L$ comes out to be $L=64 \pm 23 \mathrm{MeV}$; displayed as the shaded region of the left panel in Fig. 4. The correlation coefficients of $L$ with $\Delta r_{\mathrm{np}}$ and with $\Delta r_{\mathrm{np}}^{\text {bulk }}$ are lower than in the case of the correlations displayed in Figs. 1 and 2, suggesting that the neutron-skin thickness is slightly better correlated with $J-a_{\text {sym }}(A)$ or the ratio $a_{\text {sym }}(A) / J$ than with the slope parameter $L$. This might be a feature of the families we have chosen and does not necessarily apply to the situation in which one employs a large set of unbiasedly selected models [14]. As above, the 


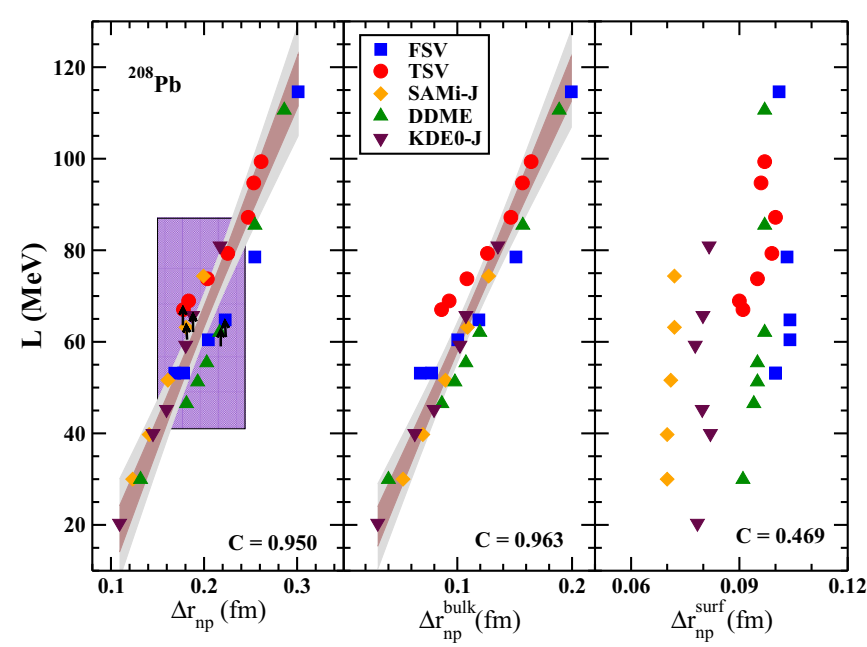

FIG. 4. Plots for the symmetry-energy slope parameter $L$ as a function of the neutron-skin thickness (left), its bulk part (middle), and its surface part (right) for the ${ }^{208} \mathrm{~Pb}$ nucleus. The shaded region in the left panel projects out the values of $L=64 \pm 23 \mathrm{MeV}$ obtained from $\Delta r_{\mathrm{np}}=0.197 \pm 0.047 \mathrm{fm}$ which, in turn, is obtained by using the empirical values of $J$ and $a_{\text {sym }}(A)$ (see also Fig. 2). The arrow marks in the left panel indicate the points with the slope parameter $L \sim 65 \mathrm{MeV}$. The values of the correlation coefficients are $C\left(L, \Delta r_{\mathrm{np}}\right)=0.950, C\left(L, \Delta r_{\mathrm{np}}^{\text {bulk }}\right)=0.963$, and $C\left(L, \Delta r_{\mathrm{np}}^{\text {surf }}\right)=$ 0.469 . The inner (outer) colored regions depict the loci of the $95 \%$ confidence (prediction) bands of the regression (see, e.g., Chap. 3 of Ref. [55]).

$\Delta r_{\mathrm{np}}-L$ correlation is weaker in comparison to the $\Delta r_{\mathrm{np}}^{\text {bulk }}-L$ correlation, in qualitative agreement with Ref. [14].

The "arrow" marks in Fig. 4 indicate the five models, each from a different family, with $L$ varying in a narrow range of $62.1 \mathrm{MeV}$ to $67.0 \mathrm{MeV}$. For these five models, there happens to be a spread in $\Delta r_{\mathrm{np}}$ of almost $0.05 \mathrm{fm}$, which is larger than expected. In comparison, the equation of the linear fit of the results of all models in the left panel of Fig. 4 gives a variation in the value of $\Delta r_{\mathrm{np}}\left({ }^{208} \mathrm{~Pb}\right)$ with the change of $L$ as $\delta\left(\Delta r_{\mathrm{np}}\right) \simeq 0.002 \delta L$, so that a change in $L$ of $5 \mathrm{MeV}$ implies an average change in $\Delta r_{\mathrm{np}}$ of about $0.01 \mathrm{fm}$ only, which is smaller than the observed spread of $0.05 \mathrm{fm}$ in the five models mentioned above. The DM supports a similar conclusion, because it can be seen from Eq. (21) that the DM predicts an average variation of $\Delta r_{\mathrm{np}}$ $\left({ }^{208} \mathrm{~Pb}\right)$ with $L$ approximately as $\delta\left(\Delta r_{\mathrm{np}}\right) \simeq 0.003 \delta L$. The two mentioned models from the TSV and SAMi-J families have $L=67 \mathrm{MeV}$ and $L=63.2 \mathrm{MeV}$, respectively, and yield in ${ }^{208} \mathrm{~Pb}$ smaller values of $\Delta r_{\mathrm{np}} \simeq 0.18 \mathrm{fm}$, whereas the two models from the FSV and DDME families have $L=64.8 \mathrm{MeV}$ and $L=62.1 \mathrm{MeV}$, respectively, and give rise to larger values of $\Delta r_{\mathrm{np}} \simeq 0.22 \mathrm{fm}$. The model from KDE0-J family with $L=65.7 \mathrm{MeV}$ yields an intermediate value of $\Delta r_{\mathrm{np}}\left({ }^{208} \mathrm{~Pb}\right) \simeq$ $0.19 \mathrm{fm}$. Actually, it comes as an intriguing fact that the extracted values of $\Delta r_{\mathrm{np}}$ differ by $\sim 0.05 \mathrm{fm}$ for the two models of the FSV and TSV families with similar $L$, although the parameters for these two families are obtained by using exactly the same kind of fitting protocol. In the next section, we aim to search for plausible interpretations for such differences in the

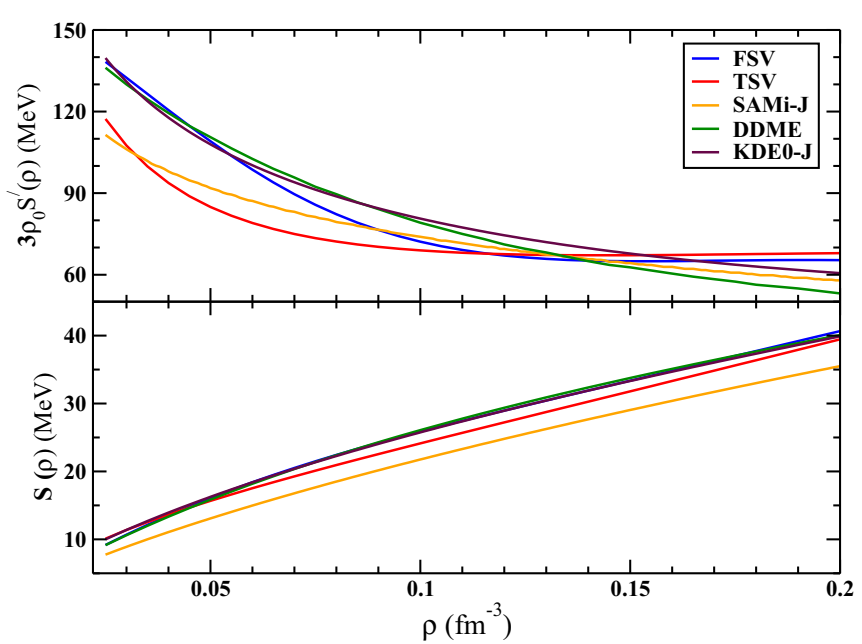

FIG. 5. The nuclear symmetry energy $S$ (lower panel) and its density derivative $S^{\prime}$ multiplied by $3 \rho_{0}$ (upper panel) as a function of density for the five different models associated with the slope parameter for nuclear matter $L \sim 65 \mathrm{MeV}$. Each of these models belongs to a different family (see also Table I).

neutron-skin thickness corresponding to models with similar $L$ values.

\section{B. Systematic differences between the families of functionals}

In an attempt to understand the issues raised at the end of the previous subsection, we make a detailed comparison between the results for the five models belonging to different families but yielding almost the same values for $L$. We first take a closer look in Fig. 5 at the values of the symmetry energy $S(\rho)$ (lower panel) and its density derivative $3 \rho_{0} S^{\prime}(\rho)$ (upper panel) as a function of density for these models. The behavior of $S(\rho)$ as a function of density seemingly appears to be similar for the five models. But the values of $3 \rho_{0} S^{\prime}(\rho)$ show significant differences in the low-density region $\left(\rho<0.10 \mathrm{fm}^{-3}\right)$. Furthermore, one may note that the TSV and SAMi-J models corresponding to $\Delta r_{\mathrm{np}}\left({ }^{208} \mathrm{~Pb}\right) \sim 0.18 \mathrm{fm}$ and the KDE0-J model with $\Delta r_{\mathrm{np}}\left({ }^{208} \mathrm{~Pb}\right) \sim 0.19 \mathrm{fm}$ display a relatively similar behavior in the density dependence of $S^{\prime}(\rho)$. The same is true for the FSV and DDME models corresponding to $\Delta r_{\text {np }}\left({ }^{208} \mathrm{~Pb}\right) \sim 0.22 \mathrm{fm}$.

To investigate whether such differences in the values of the density derivative of the symmetry energy at lower densities have an influence in the finite nuclei calculations, and motivated by Eq. (22), we determine an effective value of the slope parameter $L_{\text {eff }}$, which might be more sensitive to the relative distributions of neutrons with respect to protons in finite nuclei, as follows:

$$
L_{\mathrm{eff}}=\frac{3 \rho_{0} \int\left[r^{2} \rho(r) I^{2}(r)\right] S^{\prime}(\rho(r)) d r}{\int\left[r^{2} \rho(r) I^{2}(r)\right] d r} .
$$

Here, $I(r)$ is the local asymmetry parameter defined as, $I(r) \equiv\left[\rho_{n}(r)-\rho_{p}(r)\right] / \rho(r)$. If one assumes $S(\rho)$ to be linear in density, the $L_{\text {eff }}$ parameter coincides with $L$ [see Eq. (20)]. However, we have seen in Fig. 5 that $S(\rho)$ can depart significantly from linearity at low densities. Therefore, the $L_{\text {eff }}$ 
TABLE I. Comparison of the properties of infinite nuclear matter (NM) and of the ${ }^{208} \mathrm{~Pb}$ and ${ }^{132} \mathrm{Sn}$ nuclei for the five different models that yield a value of $L$ around $65 \mathrm{MeV}$.

\begin{tabular}{ccccccc}
\hline \hline & & SAMi-J & TSV & FSV & DDME & KDE0-J \\
\hline $\mathrm{NM}$ & $\rho_{0}\left(\mathrm{fm}^{-3}\right)$ & 0.157 & 0.147 & 0.149 & 0.152 & 0.162 \\
& $L(\mathrm{MeV})$ & 63.2 & 67.0 & 64.8 & 62.1 & 65.7 \\
& $J(\mathrm{MeV})$ & 30.00 & 31.29 & 33.16 & 34.00 & 35.00 \\
${ }^{208} \mathrm{~Pb}$ & $a_{\text {sym }}(A)(\mathrm{MeV})$ & 20.35 & 22.20 & 22.28 & 23.15 & 24.18 \\
& $\Delta r_{\text {np }}(\mathrm{fm})$ & 0.181 & 0.178 & 0.223 & 0.217 & 0.188 \\
& $\Delta r_{\text {np }}^{\text {bulk }}(\mathrm{fm})$ & 0.109 & 0.086 & 0.119 & 0.120 & 0.108 \\
& $L_{\text {eff }}(\mathrm{MeV})$ & 81.2 & 82.7 & 95.7 & 96.5 & 90.8 \\
${ }^{132} \mathrm{Sn}$ & $a_{\text {sym }}(A)(\mathrm{MeV})$ & 19.24 & 21.27 & 21.25 & 22.13 & 23.06 \\
& $\Delta r_{\text {np }}(\mathrm{fm})$ & 0.245 & 0.239 & 0.289 & 0.279 & 0.249 \\
& $\Delta r_{\text {np }}^{\text {bulk }}(\mathrm{fm})$ & 0.165 & 0.130 & 0.163 & 0.165 & 0.163 \\
& $L_{\text {eff }}(\mathrm{MeV})$ & 84.3 & 85.7 & 101.2 & 98.0 & 97.8 \\
\hline \hline
\end{tabular}

parameter as defined in Eq. (23) tries to take into account this effect. At very low densities $\left(\rho<0.01 \mathrm{fm}^{-3}\right), S(\rho)$ deviates largely from linearity. The integrals in the numerator and denominator of Eq. (23) are thus evaluated by integrating from the center of the nucleus, where the density $\rho(r)$ is of the order of $\rho_{0}$, up to the point where the density of the nucleus falls to $0.01 \mathrm{fm}^{-3}$, which corresponds to a radial coordinate $r$ of about $9 \mathrm{fm}$. It is worthwhile mentioning that we wanted to study the effect of $S^{\prime}(\rho)$ but not the quantity $L(\rho)\left[\equiv 3 \rho S^{\prime}(\rho)\right]$ on the $\Delta r_{\text {np }}$ of a heavy nucleus. That is why we kept $\rho_{0}$ outside the integral of the numerator in Eq. (23). The values of $L_{\mathrm{eff}}$ along with various other properties evaluated for the five models corresponding to $L \sim 65 \mathrm{MeV}$ are compared in Table I.

It can be easily observed in Table I that, although the values of $L$ for these models vary only by $\sim 5 \mathrm{MeV}$, the values of $\Delta r_{\mathrm{np}}$ of heavy nuclei calculated from the same models can differ by $\sim 0.05 \mathrm{fm}$, which is larger than the average spread of the correlation between $\Delta r_{\mathrm{np}}$ and $L$. Interestingly, when we look at the extracted $L_{\text {eff }}$ parameter, the models from SAMi-J and TSV families those predict $\Delta r_{\mathrm{np}}\left({ }^{208} \mathrm{~Pb}\right) \sim 0.18 \mathrm{fm}$ give similar $L_{\text {eff }} \sim 82 \mathrm{MeV}$, and the models from FSV and DDME families those predict $\Delta r_{\mathrm{np}}\left({ }^{208} \mathrm{~Pb}\right) \sim 0.22 \mathrm{fm}$ give similar $L_{\mathrm{eff}} \sim 96$ $\mathrm{MeV}$. The model from the KDE0-J family with $\Delta r_{\mathrm{np}}\left({ }^{208} \mathrm{~Pb}\right) \sim$ $0.19 \mathrm{fm}$ predicts $L_{\text {eff }} \sim 91 \mathrm{MeV}$. That is, the models with larger $L_{\text {eff }}$ give larger $\Delta r_{\mathrm{np}}$ and vice versa. In fact, further inspection of Fig. 4 reveals that two members of the FSV and DDME families with $\Delta r_{\text {np }}\left({ }^{208} \mathrm{~Pb}\right) \sim 0.18 \mathrm{fm}$, the same as the SAMi-J and TSV models in Table I, predict departing $L$ values $(L=$ 53.2 MeV in the FSV model and $L=46.5 \mathrm{MeV}$ in the DDME model). It turns out that these FSV and DDME models also explore similar values of $L_{\text {eff }}(83.9 \mathrm{MeV}$ in FSV and $86.6 \mathrm{MeV}$ in DDME) as done by the models from the SAMi-J and TSV families displayed in Table I with $\Delta r_{\mathrm{np}} \sim 0.18 \mathrm{fm}$. In principle, one can also define $L_{\text {eff }}$ without the $I^{2}(r)$ terms in Eq. (23). That is why we repeated the calculations of $L_{\text {eff }}$ by taking $I^{2}(r)$ to be unity in Eq. (23) and found similar trends as explained above. In Table I, concerning the properties of uniform matter, it is also noticeable that the models do not display the same value of the saturation density. For the nonrelativistic functionals belonging to the SAMi-J and KDE0-J family this

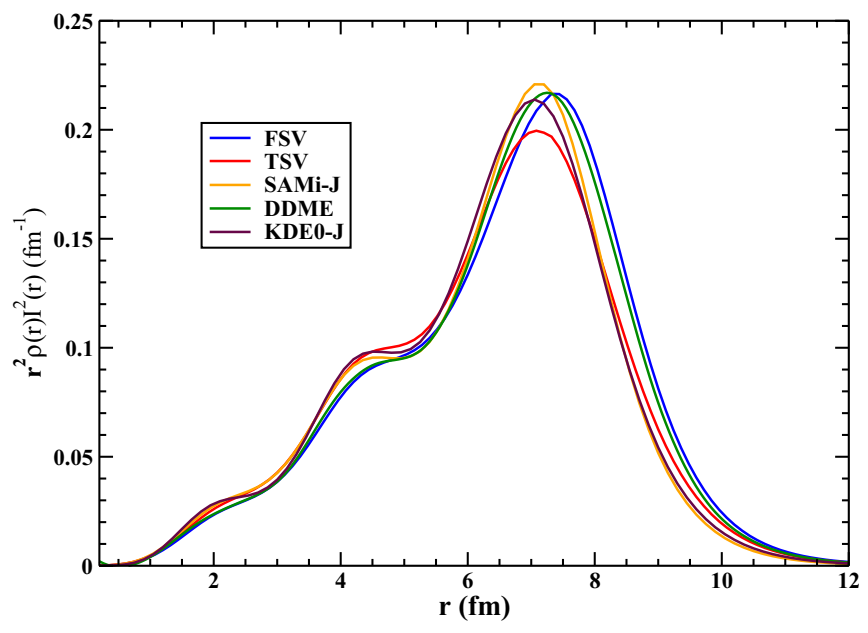

FIG. 6. The variation of $r^{2} \rho(r) I^{2}(r)$ as a function of the radial coordinate $r$ in ${ }^{208} \mathrm{~Pb}$ for the five models that yield a symmetry-energy slope parameter $L \sim 65 \mathrm{MeV}$.

value is about $5 \%-10 \%$ larger than the values explored by the relativistic functionals. This fact has some impact on the extracted values of $L_{\text {eff }}$ for these models [see Eq. (23)].

To have a better insight into the source of the differences between the values of $L_{\text {eff }}$ for the models with similar values of $L$ at $\rho_{0}$, we plot in Fig. 6 the total density distribution $\rho(r)$ of ${ }^{208} \mathrm{~Pb}$ multiplied by $r^{2} I^{2}(r)$ for the models with $L \sim$ $65 \mathrm{MeV}$. The values of $r^{2} \rho(r) I^{2}(r)$ for all the different cases are close to each other up to $r \sim 6 \mathrm{fm}$; in this region $\rho(r) \geqslant$ $0.1 \mathrm{fm}^{-3}$. With further increase in $r$, the differences in the values of $r^{2} \rho(r) I^{2}(r)$ gradually become noticeable. One can argue that different behavior in the surface region may be responsible for different values of $L_{\text {eff }}$ and consequently lead to different values of $\Delta r_{\mathrm{np}}$ in heavy nuclei such as ${ }^{208} \mathrm{~Pb}$ or

${ }^{132} \mathrm{Sn}$. The question still remains whether $L_{\text {eff }}$ is more sensitive to the density dependence of $S^{\prime}(\rho)$ (upper panel of Fig. 5) or to the density distributions of nucleons inside the nucleus (Fig. 6). To unmask this, we calculated the values of $L_{\text {eff }}$ using $S^{\prime}(\rho)$ of a given model, but with the density distributions of nucleons from the five models that have $L \sim 65 \mathrm{MeV}$. We repeated this calculation for the different choices of $S^{\prime}(\rho)$ of these five models. The values of $L_{\text {eff }}$ so obtained did not show the trend as observed in Table I, where $S^{\prime}(\rho)$ and the density distributions of nucleons used correspond to the same model consistently. Thus, the values of $L_{\text {eff }}$ are sensitive to both the density dependence of the symmetry energy and the density distributions of nucleons inside the nucleus. To this end, we would like to point out that the differences in the values of $L_{\text {eff }}$ for the models with similar $L$ parameter are mainly due to the differences in the low-density behavior of $S^{\prime}(\rho)$ and the distributions of nucleons in the surface region of the nucleus.

\section{SUMMARY}

In this work, we revisit the correlations of the neutronskin thickness in finite nuclei with various symmetry-energy parameters pertaining to infinite nuclear matter. Particular attention is paid to the model dependence in such correlations 
that can play a role in understanding the density dependence of the nuclear symmetry energy. The finite nuclei analyzed are ${ }^{208} \mathrm{~Pb}$ and ${ }^{132} \mathrm{Sn}$. The symmetry-energy parameters considered are $J-a_{\text {sym }}(A), a_{\text {sym }}(A) / J$, and $L$, where $J$ and $L$ are the symmetry energy and the symmetry-energy slope associated with infinite nuclear matter at the saturation density, and $a_{\text {sym }}(A)$ corresponds to the symmetry-energy parameter in finite nuclei. Five different families of systematically varied mean-field models corresponding to different energy density functionals are employed to calculate the relevant quantities for the finite nuclei and those for the infinite nuclear matter. Consideration of recent constraints on the symmetry-energy parameters $\left[a_{\mathrm{sym}}(A)\right.$ and $\left.J\right]$ and the present correlations suggest the values $\Delta r_{\mathrm{np}}=0.197 \pm 0.047 \mathrm{fm}$ and $\Delta r_{\mathrm{np}}=$ $0.260 \pm 0.050 \mathrm{fm}$ for the neutron-skin thickness in the ${ }^{208} \mathrm{~Pb}$ and ${ }^{132} \mathrm{Sn}$ nuclei, respectively, and $L=64 \pm 23 \mathrm{MeV}$.

In general, the correlations of the neutron-skin thickness with the different symmetry-energy parameters are strong within the individual families of the models. Once the results for all the different families are combined, the correlation coefficients become smaller, indicating a model dependence. The neutron skin in a nucleus entails two main components related to the geometry of the nucleon density profiles. On the one hand, there is a bulk contribution $\left(\Delta r_{\mathrm{np}}^{\text {bulk }}\right)$ produced by the separation between the effective sharp surfaces of the density distributions of neutrons and protons. On the other hand, there is a surface contribution $\left(\Delta r_{\mathrm{np}}^{\text {surf }}\right)$ caused by the different surface widths of the neutron- and proton-density profiles. The correlations of the symmetry-energy parameters with the bulk part $\Delta r_{\mathrm{np}}^{\text {bulk }}$ of the neutron-skin thickness are less model dependent than with the total neutron-skin thickness $\Delta r_{\mathrm{np}}$. Exceptionally, the bulk part of the neutron-skin thickness is correlated with $J-a_{\text {sym }}(A)$ and $a_{\text {sym }}(A) / J$ in an almostmodel-independent manner. This fact is much compatible with the predictions of the macroscopic droplet model.

We notice a model dependence in the correlations of the neutron-skin thickness with the symmetry-energy slope parameter $L$ when the results of the various families of models are considered together. By model dependence we mean that different models of different families with the same value of the slope $L$ of the symmetry energy predict different neutron-skin thickness, or vice versa. For different models having similar slope parameter $L \sim 65 \mathrm{MeV}$ and belonging to the different families, a spread in $\Delta r_{\mathrm{np}}$ of about $0.05 \mathrm{fm}$ is observed, which is large in view of the average spread of the correlation (Fig. 4), as well as in view of the DM estimate for the change of $\Delta r_{\mathrm{np}}$ with $L$.

We found two independent indications that the surface of the nucleus plays a key role in introducing a model dependence or, in other words, a systematic theoretical uncertainty, to the well-known linear correlation between the neutron-skin thickness and $L$ and to some other correlations that can be used to extract the parameters characterizing the density dependence of the symmetry energy. These indications are (i) the existence of stronger correlations of various symmetryenergy parameters with the bulk part of the neutron-skin thickness rather than with the total neutron-skin thickness, and (ii) the differences between the density distributions for the nucleons at the surface region for the different models corresponding to similar values of the slope parameter $L$.

To understand better the model dependence in the various correlations considered, the results are compared for the models belonging to different families but yielding similar values of $L$. We determined an effective value of the symmetry-energy slope parameter $L_{\text {eff }}$ by using the density distributions of nucleons and the density derivative of the symmetry energy for these models. It is found that the values of $\Delta r_{\mathrm{np}}$, which differ for the models with the same $L \sim 65 \mathrm{MeV}$, are in harmony with the values of $L_{\text {eff }}$. We conclude that differences in the values of $L_{\text {eff }}$ caused by differences in the density distributions of nucleons in the surface region and the derivative of the symmetry energy at subsaturation densities are the plausible sources for the aforesaid model dependence.

\section{ACKNOWLEDGMENTS}

G.C. would like to thankfully acknowledge the nice hospitality extended to him during his visit to SINP, when this work has started. N.P. acknowledges support from FP7-PEOPLE2011-COFUND program NEWFELPRO, the Croatian Science Foundation under the project Structure and Dynamics of Exotic Femtosystems (IP-2014-09-9159), and the QuantiXLie Center of Excellence. M.C. and X.V. acknowledge partial support from Grant No. FIS2014-54672-P from the Spanish MINECO and FEDER, Grant No. 2014SGR-401 from Generalitat de Catalunya, the Consolider-Ingenio 2010 Programme CPAN CSD2007-00042, and the project MDM-2014-0369 of ICCUB (Unidad de Excelencia María de Maeztu) from MINECO.
[1] S. L. Shapiro and S. A. Teukolsky, Black Holes, White Dwarfs and Neutron Stars (Wiley, New York, 1983).

[2] N. K. Glendenning, Compact Stars: Nuclear Physics, Particle Physics, and General Relativity (Springer-Verlag, New York, 2000).

[3] R. Furnstahl, Nucl. Phys. A 706, 85 (2002).

[4] P. Danielewicz, Nucl. Phys. A 727, 233 (2003).

[5] A. W. Steiner, M. Prakash, J. M. Lattimer, and P. Ellis, Phys. Rep. 411, 325 (2005).

[6] M. Centelles, X. Roca-Maza, X. Viñas, and M. Warda, Phys. Rev. Lett. 102, 122502 (2009).
[7] M. Warda, X. Viñas, X. Roca-Maza, and M. Centelles, Phys. Rev. C 80, 024316 (2009).

[8] J. Piekarewicz, B. K. Agrawal, G. Colò, W. Nazarewicz, N. Paar, P.-G. Reinhard, X. Roca-Maza, and D. Vretenar, Phys. Rev. C 85, 041302(R) (2012).

[9] B. K. Agrawal, J. N. De, and S. K. Samaddar, Phys. Rev. Lett. 109, 262501 (2012).

[10] X. Roca-Maza, M. Brenna, B. K. Agrawal, P. F. Bortignon, G. Colò, L.-G. Cao, N. Paar, and D. Vretenar, Phys. Rev. C 87, 034301 (2013). 
[11] X. Roca-Maza, M. Brenna, G. Colò, M. Centelles, X. Viñas, B. K. Agrawal, N. Paar, D. Vretenar, and J. Piekarewicz, Phys. Rev. C 88, 024316 (2013).

[12] X. Roca-Maza, M. Centelles, X. Viñas, and M. Warda, Phys. Rev. Lett. 106, 252501 (2011).

[13] M. B. Tsang et al., Phys. Rev. C 86, 015803 (2012).

[14] M. Centelles, X. Roca-Maza, X. Viñas, and M. Warda, Phys. Rev. C 82, 054314 (2010).

[15] Euro. Phys. J. A Topical Issue on Nuclear Symmetry Energy (Springer, Societa Italina di Physica, 2014), Vol. 50.

[16] L.-W. Chen, C. M. Ko, and B.-A. Li, Phys. Rev. C 72, 064309 (2005).

[17] C. Mondal, B. K. Agrawal, and J. N. De, Phys. Rev. C 92, 024302 (2015).

[18] P.-G. Reinhard and W. Nazarewicz, Phys. Rev. C 93, 051303(R) (2016).

[19] X. Roca-Maza, X. Viñas, M. Centelles, B. K. Agrawal, G. Colò, N. Paar, J. Piekarewicz, and D. Vretenar, Phys. Rev. C 92, 064304 (2015).

[20] B.-A. Li, L.-W. Chen, and C. M. Ko, Phys. Rep. 464, 113 (2008).

[21] W. D. Myers and W. J. Swiatecki, Ann. Phys. (NY) 55, 395 (1969).

[22] W. D. Myers and W. J. Swiatecki, Nucl. Phys. A 336, 267 (1980).

[23] B. A. Brown, Phys. Rev. Lett. 85, 5296 (2000).

[24] S. Typel and B. A. Brown, Phys. Rev. C 64, 027302 (2001).

[25] S. S. Avancini, J. R. Marinelli, D. P. Menezes, M. M. W. Moraes, and C. Providência, Phys. Rev. C 75, 055805 (2007).

[26] I. Vidana, C. Providencia, A. Polls, and A. Rios, Phys. Rev. C 80, 045806 (2009).

[27] W. Satuła, R. A. Wyss, and M. Rafalski, Phys. Rev. C 74, 011301 (2006).

[28] C. J. Horowitz and J. Piekarewicz, Phys. Rev. Lett. 86, 5647 (2001).

[29] S. Abrahamyan et al., Phys. Rev. Lett. 108, 112502 (2012).

[30] D. J. Dubach and I. Sick, Nucl. Phys. A 503, 589 (1989).

[31] http://hallaweb.jlab.org/parity/prex (2014).

[32] G. W. Hoffmann et al., Phys. Rev. C 21, 1488 (1980).

[33] J. Zenihiro et al., Phys. Rev. C 82, 044611 (2010).

[34] A. Krasznahorkay, H. Akimune, A. van den Berg, N. Blasi, S. Brandenburg, M. Csatlós, M. Fujiwara, J. Gulyás, M. Harakeh et al., Nucl. Phys. A 731, 224 (2004).

[35] B. Klos et al., Phys. Rev. C 76, 014311 (2007).

[36] E. Friedman et al., Hyperfine Interact. 193, 33 (2009).

[37] C. M. Tarbert et al. (Crystal Ball at MAMI and A2 Collaboration), Phys. Rev. Lett. 112, 242502 (2014).

[38] A. Tamii, I. Poltoratska, P. von Neumann-Cosel, Y. Fujita, T. Adachi, C. A. Bertulani, J. Carter, M. Dozono, H. Fujita, K. Fujita et al., Phys. Rev. Lett. 107, 062502 (2011).
[39] T. Hashimoto, A. M. Krumbholz, P.-G. Reinhard, A. Tamii, P. von Neumann-Cosel, T. Adachi, N. Aoi, C. A. Bertulani, H. Fujita, Y. Fujita et al., Phys. Rev. C 92, 031305 (2015).

[40] D. M. Rossi, P. Adrich, F. Aksouh, H. Alvarez-Pol, T. Aumann, J. Benlliure, M. Böhmer, K. Boretzky, E. Casarejos, M. Chartier et al., Phys. Rev. Lett. 111, 242503 (2013).

[41] B. A. Brown, W. A. Richter, and R. Lindsay, Phys. Lett. B 483, 49 (2000).

[42] B. D. Serot and J. D. Walecka, Adv. Nucl. Phys. 16, 1 (1986)

[43] J. Boguta and A. R. Bodmer, Nucl. Phys. A 292, 413 (1977).

[44] D. Vautherin and D. M. Brink, Phys. Rev. C 5, 626 (1972).

[45] M. Warda, X. Viñas, X. Roca-Maza, and M. Centelles, Phys. Rev. C 81, 054309 (2010).

[46] R. W. Hasse and W. D. Myers, Geometrical Relationships of Macroscopic Nuclear Physics (Springer-Verlag, Heidelberg, 1988).

[47] M. Centelles, M. Del Estal, and X. Viñas, Nucl. Phys. A 635, 193 (1998).

[48] M. Del Estal, M. Centelles, and X. Viñas, Nucl. Phys. A 650, 443 (1999).

[49] J. Liu, Z. Ren, C. Xu, and R. Xu, Phys. Rev. C 88, 024324 (2013)

[50] X. Roca-Maza, G. Colò, and H. Sagawa, Phys. Rev. C 86, 031306 (2012).

[51] D. Vretenar, T. Niksic, and P. Ring, Phys. Rev. C 68, 024310 (2003).

[52] B. G. Todd-Rutel and J. Piekarewicz, Phys. Rev. Lett. 95, 122501 (2005).

[53] M. Del Estal, M. Centelles, X. Viñas, and S. K. Patra, Phys. Rev. C 63, 024314 (2001).

[54] B. K. Agrawal, S. Shlomo, and V. K. Au, Phys. Rev. C 72, 014310 (2005).

[55] N. R. Draper and H. Smith, Applied Regression Analysis (Wiley, New York, 1981).

[56] S. Brandt, Statistical and Computational Methods in Data Analysis, 3rd English edition (Springer, New York, 1997).

[57] X. Fan, J. Dong, and W. Zuo, Phys. Rev. C 89, 017305 (2014).

[58] P. Möller, W. D. Myers, H. Sagawa, and S. Yoshida, Phys. Rev. Lett. 108, 052501 (2012).

[59] H. Jiang, G. J. Fu, Y. M. Zhao, and A. Arima, Phys. Rev. C 85, 024301 (2012).

[60] P. Danielewicz and J. Lee, Nucl. Phys. A 818, 36 (2009).

[61] A. Carbone, G. Colò, A. Bracco, L.-G. Cao, P. F. Bortignon, F. Camera, and O. Wieland, Phys. Rev. C 81, 041301(R) (2010).

[62] J. M. Lattimer and Y. Lim, Astrophys. J. 771, 51 (2013). 